\title{
Ultrastructural observations on (pseudo-) exfoliation of the lens capsule: a re-examination of the involvement of the lens epithelium
}

\author{
J. P. G. BERGMANSON, ${ }^{1}$ W. L. JONES,${ }^{2}$ AND L. W-F. CHU 1 \\ From the ${ }^{1}$ University of Houston College of Optometry, Houston, Texas 77004, and the \\ ${ }^{2}$ Department of Ophthalmology, Veterans Administration Hospital, 2100 Ridgecrest Drive SE, \\ Albuquerque, New Mexico 87108, USA
}

SUMMARY One lens each from 2 separate patients suffering from pseudoexfoliation and one lens from a normal eye were examined ultrastructurally. The normal lens capsule was internally lamellar in places and externally of a somewhat reduced electron density but otherwise of a homogeneous appearance and free of inclusions. Observations on the abnormal lenses revealed an electron dense and mainly granular but sometimes fibrillar material along the surface of the peripheral one-third of the capsule. Material of similar ultrastructural appearance was also found deeper in the capsule and in the immediately underlying epithelium. Since the capsule close to the pole of the lens was completely free of these abnormal inclusions and the underlying epithelium also lacked this material, it is concluded that the lens epithelium is a source of the surface debris in pseudoexfoliation. These findings therefore support previous authors who proposed a lenticular involvement in this disease. The present study does, however, not rule out the possibility of a uveal contribution of abnormal material but disputes its exclusive involvement in this disease, as has previously been postulated. Build-up of exfoliated material close to the capsular surface caused an apparent peeling of the lens capsule. It is therefore concluded that the anomalous lenses suffered from a true exfoliative process that may be more correctly termed 'exfoliation of the lens capsule.'

Exfoliation or pseudoexfoliation (both commonly used terms to describe this entity), along with fibrillopathia epitheliocapsularis and senile exfoliation, was first described by Lindberg' in 1917 . He described the phenomenon as consisting 'of small, greyish white or bluish grey scales which originate from the extreme pupillary margin - the scales are often very small and appear as bluish grey dust particles.' Lindberg also noticed that $50 \%$ of his patients with exfoliation had glaucoma and $20 \%$ had cataracts. Vogt $^{2}$ in 1931 was the first to coin the term 'glaucoma capsulare' as the glaucomatous condition accompanied by exfoliation. In 1954 Dvarak-Theobald $^{3}$ introduced the term 'pseudoexfoliation of the lens capsule.'

Exfoliation was first noticed in the Scandinavian countries, where it was believed to have a much higher incidence than elsewhere. A study by Horven ${ }^{4}$

Correspondence to Dr J. P. G. Bergmanson, College of Optometry, University of Houston, Houston, Texas 77004, USA. in 1939 , noted an 80 to $90 \%$ incidence of primary open-angle glaucoma with exfoliation in Norway. However, this was at a referral centre in Norway for glaucoma and reflected a much higher incidence than in the normal population, since more difficult cases to treat are referred. There is a much higher incidence of open-angle glaucoma in patients with exfoliation which is often a more treatment-resistant type of open-angle glaucoma. ${ }^{5-7} \mathrm{~A}$ later study of glaucoma capsulare revealed a $32.9 \%$ occurrence in patients with open-angle glaucoma in Norway. ${ }^{8}$ Studies of the general Scandinavian population revealed between 2 and $8 \%$ prevalence of exfoliation. ${ }^{6910}$ The incidence of exfoliation in glaucoma clinics in the United States was reported to be $28 \%,{ }^{11} 12 \%,{ }^{12} 7 \%,{ }^{13} 3 \cdot 7 \%,{ }^{14}$ and $2 \% .{ }^{15}$ The prevalence of exfoliation in patients over 60 years of age is $6.3 \%$ in Norway, $4 \%$ in Germany, and $4.7 \%$ in England. ${ }^{6}$ There was a $7.2 \%$ prevalence in the United States for all ages. ${ }^{16}$ Grable and Sugar ${ }^{17}$ found exfoliation to be rare in American Negroes, 
but it has a high incidence in the Bantu-speaking tribes of South Africa. ${ }^{18}$ However, it appears to be present in all racial groups more or less in proportion to their population. ${ }^{19}$ Originally, geography was proposed as a predisposing factor, but exfoliation has been found in many areas of the world and in many different types of environments. ${ }^{61820-26}$

The older the age group studied, the greater the incidence of exfoliation. It is more commonly found in patients 60 years and above, ${ }^{27}$ with the highest discovery rate in the 60 to 69 age group, and the average age seems to be around 69 to 73 years. ${ }^{132}$ It is rare under the age of 50 years, ${ }^{29}$ and the youngest reported case was only 31 years of age.$^{30}$ The number of cases decreases in the 70s and $80 \mathrm{~s}$, but this is a reflection of an increase in mortality rates. Published reports state that females have a greater incidence, others that males do, and some state that there is no difference. ${ }^{8131627}$ There is no predilection for the right or left eye in most reported cases.

Exfoliation material has been found to be produced in the lens, ciliary body, iris, and the outer wall of conjunctival vessels. ${ }^{5132}$ Exfoliative material can be found deposited on other structures in the eye such as the zonules, anterior vitreous face, posterior capsule, pupillary border, anterior iris surface, posterior corneal surface, and in the anterior chamber angle. However, some controversy exists over where the source of the exfoliative material found on the lens surface is exactly located. A number of earlier studies $^{33-35}$ indicated the lens epithelium as the source, while other reports ${ }^{36-39}$ disputed that theory by suggesting that the material is of uveal origin and only deposited on the lens as opposed to originating from it.

The purpose of this study was to examine ultrastructurally the human crystalline lens the better to understand the mechanism behind this disease process of the eye, and to re-evaluate the possible involvement of the lens epithelium.

\section{Material and methods}

Two lenses with pseudoexfoliation from 2 different patients and one normal human control lens were examined electron microscopically.

Immediately after extraction the cataractous lenses with exfoliation were placed in fixative $(3 \%$ glutaraldehyde in $0 \cdot 1 \mathrm{M}$ cacodylate buffer at $\mathrm{pH} 7 \cdot 2$ ). The control lens obtained from the eye bank at the University of Texas Medical School in Houston was submersed in similar fixative. The exfoliated and control lenses were subsequently dissected and processed along identical protocols.

The lenses were bisected equatorially, and the anterior half was further divided into equatorial, mid- peripheral, and central portions. The material was selected from random meridional orientations and amounted to between a $1 / 4$ and $1 / 8$ of the anterior lens.

The tissues were first washed in $0 \cdot 1 \mathrm{M}$ cacodylate buffer at $\mathrm{pH} 7.2$ and then stained in $1 \%$ osmium tetroxide in $0.1 \mathrm{M}$ cacodylate buffer at $\mathrm{pH} 7.2$ for 3 hours. After dehydration of the material through an alcohol series it was embedded in Spurr's lowviscosity embedding medium. Areas to be used for thin sections were selected from light microscopic observations of semithin $(1 \mu \mathrm{m})$ toluidine blue stained sections. The thin sections were stained for approximately 10 minutes in each of the $3.5 \%$ uranyl acetate and Reynolds's lead citrate solutions. The preparations were examined in a Jeol JEM-100C electron microscope.

\section{Results}

The lens fibres and epithelium were enclosed externally by a basement membrane. This basement membrane, termed the lens capsule, was normally relatively homogeneous in appearance and contained no dense granular matter (Figs. 1, 2 inset). However, a distinctly lamellar pattern was noted periodically in the internal aspect of the capsule (Fig. 3). Thin empty spaces were apparent between these lamellae. Close to the external surface of the capsule a reduced electron density was observed in places (Fig. 2 inset).

Towards the equator the cuboidal epithelial cells started elongating, but the cytoplasmic content appeared unchanged (Fig. 1). Apart from a spherical nucleus the cytoplasm also contained moderate numbers of mitochondria and rough and smooth endoplasmic reticulum. The anterior plasmalemma and to a lesser degree also the lateral wall showed deep invaginations. Widened invaginations of the anterior plasmalemma produced epithelial pockets, where the open end faced the lens capsule (Fig. 2). Tight junctions were observed between cells along the external third of the lateral cell wall (Fig. 2).

Internal to the lens epithelium the lens fibres were densely packed, leaving no interstitial spaces (Fig. 1). The fibres were held together by desmosomes and characteristic ball and socket joints. A plasmalemma surrounded the fibres, which lacked organelles.

The anterior epithelium in the exfoliated lenses showed an increase in size and numbers of empty spaces along its full length (Fig. 4). Approximately half way towards the equator sporadic fibrillar and granular structures appeared in the epithelium and the capsule. Two-thirds of the way this material became a significant to dominant part of the anterior lens morphology (Figs. 4 and 5). The capsule appeared to be peeling off owing to the accumulation 

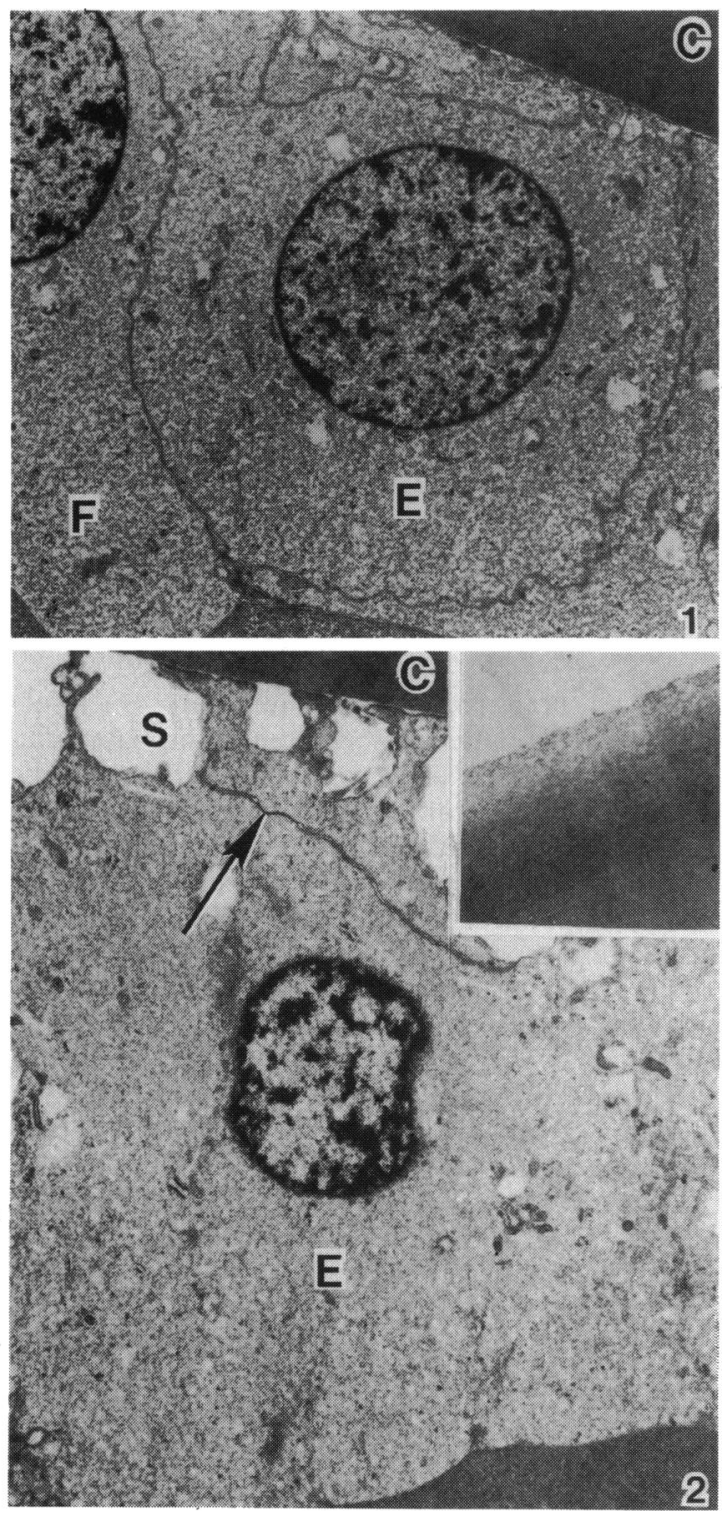

of exfoliative material beneath the capsular surface (Fig. 4 inset).

The granular substance appeared to be distributed over much of the cytoplasm of the cell, while the fibrillar material was found along the cell surface. At intervals the fibrillar material appeared to erupt from the external cell wall into the capsule, where this substance could be traced to the anterior surface of the lens (Fig. 5). Towards the surface this product seemed to become increasingly granular, and along the surface aggregates of the material appeared to be breaking off from the lens (Fig. 5 inset).

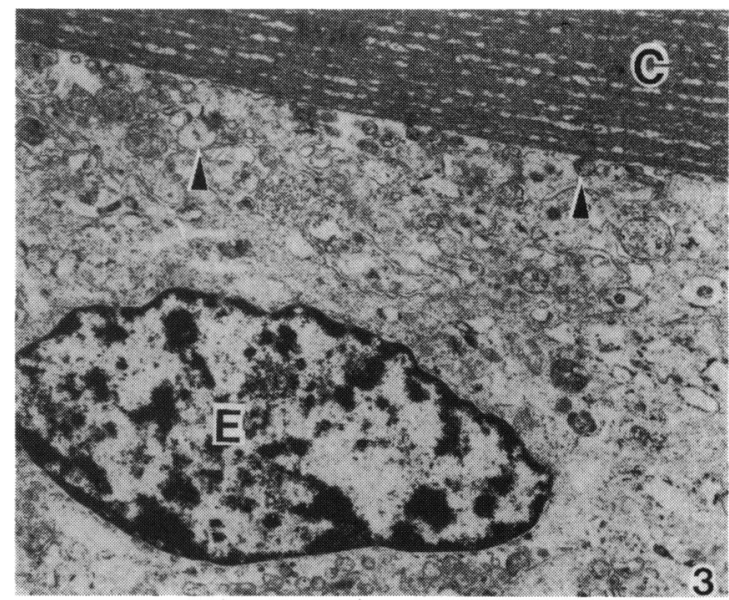

Fig. 1 Control lens. Normal lens epithelium close to the equator. The epithelium is formed by typically cuboidal cells (E) with a rounded nucleus. One cell (F) shows an elongation which is a characteristic of the equatorial region. The cytoplasm contains a normal organelle population and a uniform electron density. Externally the epithelium is covered by the relatively homogeneous lens capsule (C). $(\times 4845)$.

Fig. 2 Control lens. Normal epithelium from the midperiphery of the lens. Empty spaces $(\mathrm{S})$ are present between the epithelium (E) and the capsule (C). A tight junction (arrow) between 2 cells is close to the external surface of the epithelium. Apart from ribosomes the epithelium lacks dense granular material, while the capsule is of a homogeneous appearance. $(\times 4590)$. Inset: Control lens $(\times 6800)$ : The external capsule. The normal lens capsule contained no granular elements but a decrease in electron density of the capsule is seen in the most external region.

Fig. 3 Control lens. Junctional region between the lens capsule and the epithelium. The capsule (C) has a markedly lamellar pattern. Externally the lens epithelium (E) has developed numerous small processes (triangles). $(\times 9180)$.

\section{Discussion}

The findings on the normal lens morphology in the present study were in agreement with earlier observations..$^{40}$ The open epithelial invaginations, anteriorly orientated and towards the capsule, have been described recently also in the normal rabbit lens. ${ }^{41}$ Pitts et al. suggested that these pockets represented a normal physiological level of oedema in the lens epithelium. ${ }^{41}$ They showed that ultraviolet radiation damage to the lens had the effect of increasing this oedema. It is possible that the 


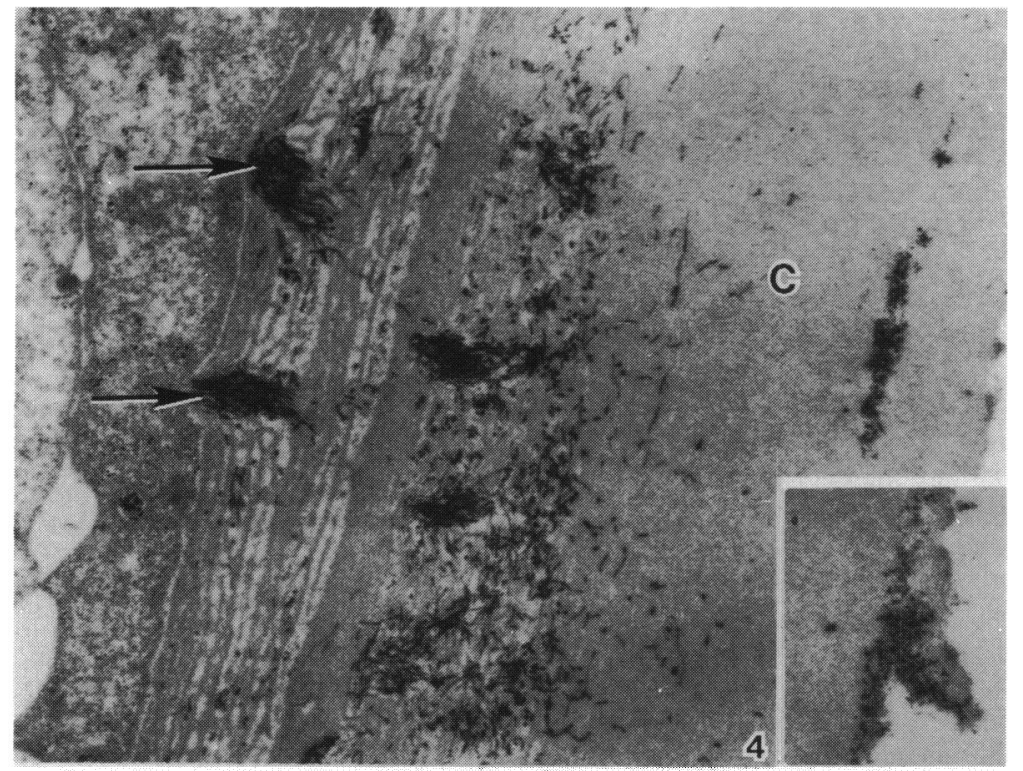

Fig. 4 Exfoliated material in the external lens. Fibrillar and granular material radiate from the surface (arrow) of the epithelial cell. The material is distributed throughout the thickness of the capsule (C) with most of it in the middle third. Exfoliated lens $(\times 9450)$. Inset: Exfoliated material along the capsular surface. Note that the capsule itself appears to be peeling off owing to the accumulation of exfoliation material beneath the capsular surface. The granular exfoliation material appears to be loosely attached to the surface of the lens. Exfoliated lens. $(\times 9450)$.

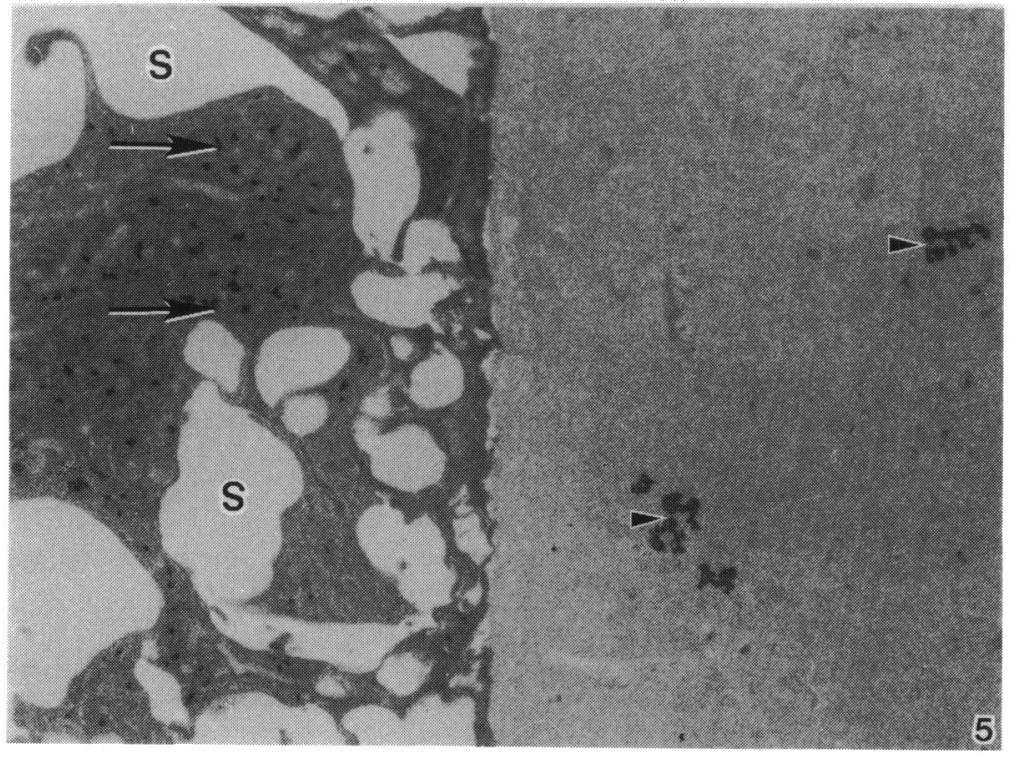

Fig. 5 Exfoliation substance in the junctional region between the lens capsule and the lens epithelium. The epithelial cytoplasm contains a dense granular substance (arrow) which is also present in the capsule (triangle). Numerous spaces (S) are present within the epithelium, while the internal capsule lacks a marked lamellar arrangement. Exfoliated lens. $(\times 9750)$.

enlarged spaces encountered in the lens epithelium of the cataractous lenses were a result of the inability of these cells to maintain normal lenticular hydration.

The lens epithelium in the lenses with exfoliation lacked abnormal cell contents centrally, while towards the equator fibrillar and granular substances were increasingly found. It was also only in the peripheral regions that abnormal and presumably exfoliative material was observed within and along the surface of the lens capsule. This observation supports earlier reports ${ }^{33-35}$ which suggested that the lens epithelium is the source of the material found along the lens surface in patients with exfoliation. Similar material has been shown to be produced elsewhere in the eye..$^{51132{ }^{36-39}}$ Studies demonstrating abnormal basement membrane material in the uvea and particularly the iris $^{36-39}$ postulated that this material was deposited on the lens through the intimate relationship between the lens and the iris in this region. However, the present study presents evidence of definite lenticular involvement in areas of capsular 
surface debris. Similarly no fibrillar or granular material was present in the epithelium where the overlying capsule was free of exfoliation material. It is nevertheless possible that the uvea contributes in this disease process. Extraocular sites of production of exfoliation material have been demonstrated, ${ }^{213142}$ suggesting that the disease is more general than initially realised. Indeed the broader term 'basement membrane exfoliation syndrome' has been proposed. ${ }^{42}$

A degenerative band within the capsule has been described as occurring in the affected region of the lens. ${ }^{3643}$ This band occupies the internal half of the capsule and contains the fibrillar exfoliation material. The exfoliated lenses examined in the present study showed a similar dense aggregation of fibrillar material in the capsule immediately adjacent to the epithelium anterior to the equator. This picture was accentuated by the tendency of the capsule to become lamellar in this region. Since the lamellar formations of the capsule were also observed in the normal lens it may be assumed that only the exfoliation material was associated with the disease, while the lamellae are possibly related to the age of the individual.

The exfoliation material is generally described as being fibrillar in nature. This was found to be the case also in the present study, especially deeper in the capsule. However, the chemical composition of these fibrils is still not fully settled. Ringvold and Husby ${ }^{44}$ suggested in a histochemical study that the exfoliation material was related to the amyloid material, while Dark et al. ${ }^{45}$ argued that, although they were rather similar, there were also differences between these substances. The latter authors postulated that 'the pre-equatorial epithelial cells synthesize long-chain zone-like proteins, which are then converted by lysozomal enzymes into small molecules capable of polymerizing both within the capsule and in relation to nearby aqueous-bathed structures.'

There has been deliberation over what name best describes this entity-'pseudoexfoliation' or 'exfoliation.' Exfoliation means to cast off in scales, laminae, or splinters or to come off in thin pieces, and it can be plainly seen in the Fig. 4 inset that the disease described here is indeed an exfoliative process. The peeling of the lens capsule appeared to be due to a build-up of exfoliative material beneath it. If one classifies 'true exfoliative' as an actual peeling of the lens capsule, it would appear that this entity fulfils that requirement. We feel that the best terminology for this entity is 'exfoliation of the lens capsule,' since it would seem to entail an actual loss of lens capsule, along with the shedding of abnormal exfoliative material from the capsular surface.

We thank Mr Tommy C. Yee for providing valuable photographic assistance.

\section{References}

1 Lindberg JG. Kliniska Undersokningar over depigmentering av pupillranden och genomlysbarhet av iris fall av alderstarr samt $i$ normala ogon hos gamla personer Diss. Helsingfors, 1917.

2 Vogt A. Lebrhuch und atlas der Spaltlampenmikroskopia des lebenden Auges. Berlin: Springer, 1931: 2: 527-93.

3 Dvorak-Theobald G. pseudoexfoliation of the lens capsule. Relation to 'true' exfoliation of the lens capsule as reported in the literature and role in the production of glaucoma capsulocuticulare. Am J Opthalmol 1954; 37: 1-12.

4 Horven E. Exfoliation of the lens capsule (Vogt) and its relation to glaucoma simplex. Br J Ophthalmol 1937; 21: 625-37.

5 Layden WE, Shaffer RN. The exfoliation syndrome. Trans Am Acad Ophthalmol Otolaryngol 1974; 78: 326-7.

6 Aasved $\mathrm{H}$. The geographical distribution of fibrillopathia epitheliocapsularis. Acta Ophthalmol (Kbh) 1969; 47: 792-810.

7 Gradle HS, Sugar HS. Concerning the chamber angle. II. Exfoliation of the zonular lamella and glaucoma capsulare. Am J Ophthalmol 1940; 23: 982-97.

8 Aasved $\mathrm{H}$. The frequency of fibrillopathia, epitheliocapsularis (so-called senile exfoliation or pseudoexfoliation) in patients with open angle glaucoma. Acta Ophthalmol (Kbh) 1971; 49: 194-210.

9 Ladekarl S. Incidence in Denmark of the so-called senile exfoliation of the lens capsule. Acta Ophthalmol (Kbh) 1965; 43: 539-42.

10 Klauman OF. Pseudoexfoliation in ophthalmic practice. Acta Ophthalmol (Kbh) 1967; 45: 822-8.

11 Horven I. Exfoliation syndrome. Arch Ophthalmol 1966; 76: 505-11.

12 Roth M, Epstein DL. Exfoliation syndrome. Am J Ophthalmol 1980; 89: 477-81.

13 Hogart DM, Yanoff M. Intraocular pressure status in 100 consecutive patients with exfoliation syndrome. Ophthalmology 1982; 89: 214-8.

14 Lemoine AN Jr. Glaucoma. A statistical review of 816 patients with 1112 glaucomatous eyes. Am J Ophthalmol 1950; 33: 1353-72.

15 Holst JC. A statistical study of glaucoma. Am J Ophthalmol 1947; 30: $1267-75$.

16 Hiller R, Sperduto RD, Krueger DE. Pseudoexfoliation, intraocular pressure, and senile lens changes in a population based survey. Arch Ophthalmol 1982; 100: 1080-2.

17 Grade HS, Sugar S. Glaucoma capsulare. Am J Ophthalmol 1947; 30: 12-9.

18 Bartholomew RS. Pseudocapsular exfoliation in the Bantu of South Africa. II. Occurrence and prevalence. Br J Ophthalmol 1973; 57: 41-5.

19 Gillies WE. Racial incidence of pseudoexfoliation of the lens capsule. Br J Ophthalmol 1972; 56: 474-7.

20 Taylor HR. Pseudoexfoliation, environmental disease. Trans Ophthalmol Soc UK 1979; 99: 302-7.

21 Bartholomew RS. Incidence of pseudoexfoliation in South African Negroes and Scots. Trans Ophthalmol Soc UK 1979; 99: 299-301.

22 Good NN. Prevalence of pseudoexfoliation of the lens capsule in India. Acta Ophthalmol (Kbh) 1968; 46: 211-4.

23 Taylor HR, Hollows FC, Maran D. Pseudoexfoliation of the lens in Australian aborigines. Br J Ophthalmol 1969; 61: 473-5.

24 Clements DB. Glaucoma in the Isle of Man with special reference to pseudocapsular exfoliation. Br J Ophthalmol 1968; 52: 546-9.

25 Aasved H. Study of relatives of persons with fibrillopathia epitheliocapsularis (pseudoexfoliation of the lens capsule). Acta Ophthalmol (Kbh) 1975; 53: 879-86.

26 Tarkkanen A, Voipio H, Koivusalo P. Family study of pseudoexfoliation and glaucoma. Acta Ophthalmol (Kbh) 1965; 43: 679-83.

27 Aasved $\mathrm{H}$. Mass screening for fibrillopathia expitheliocapsularis, so-called senile exfoliation or pseudoexfoliation of the anterior lens capsule. Acta Ophthalmol (Kbh) 1971; 49: 334-43. 
28 Luntz MH. Prevalence of pseudoexfoliation syndrome in an Urban South African clinic population. Am J Ophthalmol 1972; 74: 581-7.

29 Forsius $\mathrm{H}$. Pseudoexfoliation of the anterior capsule of the lens in Lapps and Eskimos. Can J Ophthalmol 1973; 8: 274-7.

30 Horven I, Hutchinson BT. Exfoliation syndrome: Case reports of 31 and 35 year old patients. Acta Ophthalmol (Kbh) 1967; 45: 294-8.

31 Ringvold $A$. The occurrence of pseudoexfoliation material in extrabulbar tissue from patients with pseudoexfoliation syndrome of the eye. Acta Ophthalmol (Kbh) 1973; 51: 411-8.

32 Speakman JS, Ghosh M. The conjunctiva in senile lens exfoliation. Arch Ophthalmol 1976; 94: 1750-9.

33 Bertelsen TI, Drablos PA, Flood PR. The so-called senile exfoliation (pseudoexfoliation) of the anterior lens capsule, a product of the lens epithelium. Fibrillopathis epitheliocapsularis. Acta Ophthalmol (Kbh) 1964; 42: 1096-113.

34 Seland JH. Histopathology of the lens in fibrillopathia epitheliocapsularis (FEC) on so-called senile exfoliation or pseudoexfoliation. An electron microscopic study. Acta Ophthalmol (Kbh) 1979; 57: 477-99.

35 Dickson DH, Ramsey MS. Symposium on pseudocapsular exfoliation and glaucoma. Fibrillopathia epitheliocapsularis. Review of the nature and origin of pseudoexfoliative deposits. Trans Opthalmol Soc UK 1979; 99: 284-92.

36 Ashton N, Shakib M, Collyer R, Black R. Electron microscopic study of pseudoexfoliation of the lens capsule. Invest Ophthalmol Visual Sci 1965; 4: 141-53.
37 Benedikt O, Aubock L, Gottinger W, Waltinger $\mathrm{H}$. Vergleichende rasterelektronenmikroskopische und transmissionselektronenmikroskopische Untersuchungen an Linsen bei sogenannten Exfoliationsyndrome. Albrecht von Graefes Arch Klin Ophthalmol 1973; 187: 249-64.

38 Benedikt $\mathrm{O}$. Morphologische Veranderungen beim sogenannten Exfoliationsyndrome. Klin Monatsbl Augenheilkd 1973; 162: 465-77.

39 Takei Y, Mizuno K. Electron-microscopic study of pseudoexfoliation of the lens capsule. Albrecht von Graefes Arch Klin Ophthalmol 1978; 205: 213-20.

40 Hogan MJ, Alvarado JA, Weddell JE. Histology of the human eye. An atlas and textbook. Philadelphia, London, Toronto: Saunders, 1971: 638-77.

41 Pitts DG, Bergmanson JPG, Chu LW-F. Rabbit eye exposure to broad-spectrum fluorescent light. Report to Illuminating Engineering Institute. 1982.

42 Eagle RC, Font RL, Fine BS. The basement membrane exfoliation syndrome. Arch Ophthalmol 1979; 97: 510-5.

43 Luntz MH. Exfoliation and pseudoexfoliation of the lens. In: Bellows JG, ed. Cataract and abnormalities of the lens. New York, San Francisco, London: Grune and Stratton, 1975: 344-56.

44 Ringvold A, Husby G. Pseudo-exfoliation material-an amyloid-like substance. Exp Eye Res 1973; 17: 289-99.

45 Dark AJ, Streeten BW, Cornwall CC. Pseudoexfoliative disease of the lens: a study in electron microscopy and histochemistry. $\mathrm{Br}$ J Ophthalmol 1977; 61: 462-72. 